\title{
Lipschitz stability estimate in the inverse Robin problem for the Stokes system
}

\author{
Anne-Claire Egloffe
}

August 17, 2018

\begin{abstract}
We are interested in the inverse problem of recovering a Robin coefficient defined on some non accessible part of the boundary from available data on another part of the boundary in the nonstationary Stokes system. We prove a Lipschitz stability estimate under the a priori assumption that the Robin coefficient lives in some compact and convex subset of a finite dimensional vectorial subspace of the set of continuous functions. To do so, we use a theorem proved by L. Bourgeois which establishes Lipschitz stability estimates for a class of inverse problems in an abstract framework.
\end{abstract}

Résumé Estimation de stabilité Lipschitzienne pour le système de Stokes avec des conditions aux limites de types Robin

Nous nous intéressons à l'identification d'un coefficient de Robin défini sur une partie non accessible du bord à partir de mesures disponibles sur une autre partie du bord dans le système de Stokes non stationnaire. Nous prouvons une estimation de stabilité Lipschitzienne sous l'hypothèse a priori que le coefficient de Robin est défini dans un sous-ensemble compact et convexe d'un sous-espace vectoriel de dimension finie de l'espace des fonctions continues. Pour ce faire, nous utilisons un théorème prouvé par L. Bourgeois permettant d'établir des inégalités de stabilité Lipschitzienne pour une classe de problèmes inverses dans un cadre abstrait.

\section{Version française abrégée}

Soit $T>0, \Omega \subset \mathbb{R}^{d}$, avec $d \in \mathbb{N}^{*}$, un ouvert borné Lipschitzien et connexe tel que $\partial \Omega=\Gamma_{l} \cup \Gamma_{0} \cup \Gamma_{\text {out }}$ avec $\Gamma_{\text {out }}=\bigcup_{i=1}^{N} \Gamma_{i}$ et $\nu$ est la normale extérieure à $\Omega$. On considère le système de Stokes suivant :

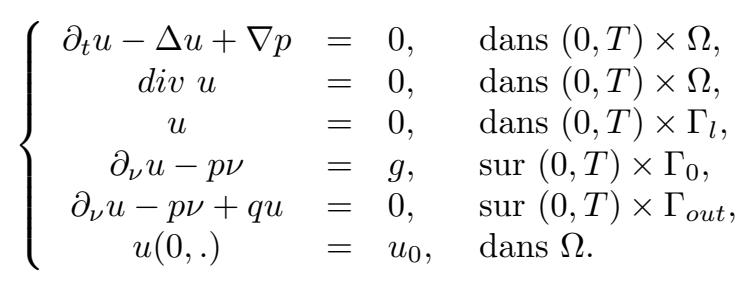

Le problème inverse qui nous intéresse est le suivant : on cherche à identifier le coefficient de Robin $q$ défini sur la partie non accessible du bord $\Gamma_{\text {out }}$ à partir de mesures disponibles sur $\Gamma_{0}$ pour $(u, p)$ solution du système (11). Ce type de problème inverse apparaît naturellement dans la modélisation d'écoulements biologiques, comme par exemple l'écoulement sanguin dans le système cardiovasculaire (voir [11] et 14]) ou encore l'écoulement de l'air dans les poumons (voir [2]). Nous renvoyons à [8] pour une introduction à la modélisation de l'écoulement de l'air dans les poumons et aux différentes conditions aux limites qui peuvent être prescrites. La résolution de ce type de problème inverse dans le cas stationnaire a déjà été étudiée dans [5], 4] et [9]. Dans les deux premiers travaux, une inégalité de stabilité logarithmique est obtenue alors qu'une inégalité de stabilité Lipschitzienne est établie dans [9] sous l'hypothèse a priori que le coefficient de Robin est constant par morceaux sur $\Gamma_{\text {out }}$. Dans chacun de ces papiers, les mesures 
intervenant dans les inégalités de stabilité sont la vitesse $u$, la pression $p$ et la dérivée normale de la pression $\frac{\partial p}{\partial n}$ sur $\Gamma \subseteq \Gamma_{0}$. Le cas du système de Stokes non stationnaire a été abordé dans [5] dans le cas particulier où le coefficient de Robin ne dépend pas du temps. L'idée, introduite dans [3] dans le cas de l'équation de Laplace, consiste à étendre l'inégalité de stabilité valable pour le problème stationnaire au problème non stationnaire en utilisant une inégalité provenant de la théorie des semigroupes analytiques. Cela conduit à faire des mesures en temps infini.

L'originalité de l'inégalité de stabilité Lipschitzienne présentée dans cette Note est multiple : d'une part, nous obtenons une inégalité de stabilité valable pour le système de Stokes non stationnaire en temps fini avec un coefficient de Robin dépendant du temps et d'autre part, l'unique mesure intervenant dans l'inégalité de stablité est la vitesse $u$ sur $(0, T) \times \Gamma$, avec $\Gamma \subseteq \Gamma_{0}$. De plus, l'ensemble des coefficients de Robin pour lequel l'inégalité de stabilité Lipschitzienne est valide est un peu plus général que dans [9] : les coefficients de Robin ne sont plus nécessairement constants par morceaux mais appartiennent à un sous-ensemble compact et convexe d'un sous-espace vectoriel de dimension finie de l'ensemble des fonctions continues. Enfin, nous avons besoin d'hypothèses de régularité moins fortes sur le bord du domaine $\Omega$ et sur le flux $g$.

Afin d'être plus précis, nous introduisons quelques notations.

Notation 1.1. On note

$$
L_{+}^{\infty}\left((0, T) \times \Gamma_{\text {out }}\right)=\left\{q \in L^{\infty}\left((0, T) \times \Gamma_{\text {out }}\right) ; \exists m>0, q \geq m p . \text { p. sur }(0, T) \times \Gamma_{\text {out }}\right\},
$$

et

$$
\mathcal{C}^{0}\left(0, T ; \mathcal{C}_{p c}^{0}\left(\Gamma_{\text {out }}\right)\right)=\left\{q:(0, T) \times \Gamma_{\text {out }} \rightarrow \mathbb{R} ;\left.q\right|_{(0, T) \times \Gamma_{i}} \in \mathcal{C}^{0}\left((0, T) \times \Gamma_{i}\right) \text { pour } 1 \leq i \leq N\right\}
$$

Le résultat principal de cette Note est résumé dans le théorème suivant :

Théorème 1.2. Soit $M \in \mathbb{N}^{*}$. On considère $V_{M}$ un sous-espace vectoriel de $\mathcal{C}^{0}\left(0, T ; \mathcal{C}_{\text {pc }}^{0}\left(\Gamma_{\text {out }}\right)\right)$ engendré par $M$ fonctions linéairement indépendantes et $K_{M}$ un sous-espace convexe et compact de $V_{M} \cap L_{+}^{\infty}\left((0, T) \times \Gamma_{\text {out }}\right)$. Soit $\Gamma \subseteq \Gamma_{0}$ une partie ouverte non vide du bord du domaine, $u_{0} \in H^{1}(\Omega)$ tel que div $u_{0}=0$ dans $\Omega$ et $g \in H^{1}\left(0, T ; L^{2}\left(\Gamma_{0}\right)\right)$ tel que $g(t)$ est non identiquement zéro pour tout $t \in(0, T)$. Soit $\left(u_{k}, p_{k}\right)$ la solution faible du système (10) avec $q=q_{k} \in K_{M}$ pour $k=1,2$. Alors, il existe une constante $C>0$ telle que

$$
\left\|q_{1}-q_{2}\right\|_{L^{\infty}\left((0, T) \times \Gamma_{\text {out }}\right)} \leq C\left\|u_{1}-u_{2}\right\|_{L^{2}((0, T) \times \Gamma)}
$$

La preuve du Théorème 1.2 est basée sur un théorème abstrait prouvé par L. Bourgeois dans [6] que nous rappelons dans le Théorème 3.4 et repose sur le fait que l'application

$$
\begin{aligned}
T: L_{+}^{\infty}\left((0, T) \times \Gamma_{\text {out }}\right) & \rightarrow L^{2}((0, T) \times \Gamma) \\
q & \left.\rightarrow u\right|_{\Gamma}
\end{aligned}
$$

où $(u, p)$ est solution du système (1) et avec $\Gamma \subseteq \Gamma_{0}$, est injective, de classe $\mathcal{C}^{1}$ et sa dérivée est également injective. Notons que le résultat énoncé dans [6] permet d'établir des inégalités de stabilité Lipschitziennes pour une classe de problèmes inverses. Il permet notamment de retrouver les résultats de stabilité développés dans [12] et [1] sans avoir recours à des arguments de quantification de résultats de continuation unique. L'auteur précise que l'on peut trouver l'idée originale développée dans [7] dans le cas particulier de la détection d'un obstacle se déplaçant dans un fluide à partir de mesures disponibles sur le bord du domaine. De plus, des théorèmes abstraits du même type mais avec des hypothèses différentes peuvent être trouvés dans [13].

\section{Introduction}

Let $T>0, \Omega \subset \mathbb{R}^{d}$, with $d \in \mathbb{N}^{*}$, be a Lipschitz bounded connected open set such that $\partial \Omega=\Gamma_{l} \cup \Gamma_{0} \cup \Gamma_{\text {out }}$ and $\Gamma_{\text {out }}=\bigcup_{i=1}^{N} \Gamma_{i}$. We are interested in the inverse problem of identifying the Robin coefficient $q$ defined on some non accessible part of the boundary $\Gamma_{\text {out }}$ from available data on $\Gamma_{0}$ for $(u, p)$ solution of the Stokes system (1). Such kinds of systems naturally appear in the modeling of biological problems like, for example, blood flow in the cardiovascular system (see [11] and [14]) or airflow in the lungs (see [2]). For an introduction on the modeling of the airflow in 
the lungs and on different boundary conditions which may be prescribed, we refer to [8]. Similar inverse problems have already been studied in the stationary case in [5], [4] and [9]. In [5] and [4], a logarithmic stability estimate is obtained, whearas a Lipschitz stability estimate is established in [9] under the a priori assumption that the Robin coefficient is piecewise constant on $\Gamma_{\text {out }}$. In each cases, the measurements involved in the stability estimates are the velocity $u$, the pressure $p$ and the normal derivative of the pressure $\frac{\partial p}{\partial n}$ on $\Gamma \subseteq \Gamma_{0}$. The case of the nonstationary Stokes system has been addressed in [5] in the particular case where the Robin coefficient does not depend time. The idea, introduced in [3] in the case of the Laplace equation, is to extend the stability estimate valid for the stationary problem to the nonstationary problem by using an inequality from the theory of analytic semigroups. This leads to infinite time measurements.

The originality of the Lipschitz stability estimate presented in this Note is multiple: on the one hand, we obtain a stability estimate valid for the nonstationary Stokes system in finite time with a time-dependent Robin coefficient and secondly, the only measurement involved in the stability estimate is the velocity $u$ on $(0, T) \times \Gamma$, with $\Gamma \subseteq \Gamma_{0}$. In addition, the set of admissible Robin coefficients is more general than in [9]: Robin coefficients are not necessarily piecewise constant but belong to some compact and convex subset of a finite dimensional vectorial subspace of the set of continuous functions. Finally, we relax the regularity assumptions needed both on the boundary of the domain $\Omega$ and on the flux $g$.

To be more precise, we introduce some notations.

Notation 2.1. We denote by

$$
L_{+}^{\infty}\left((0, T) \times \Gamma_{\text {out }}\right)=\left\{q \in L^{\infty}\left((0, T) \times \Gamma_{\text {out }}\right) ; \exists m>0, q \geq m \text { a. e. on }(0, T) \times \Gamma_{\text {out }}\right\},
$$

and

$$
\mathcal{C}^{0}\left(0, T ; \mathcal{C}_{\text {pc }}^{0}\left(\Gamma_{\text {out }}\right)\right)=\left\{q:(0, T) \times \Gamma_{\text {out }} \rightarrow \mathbb{R} ;\left.q\right|_{(0, T) \times \Gamma_{i}} \in \mathcal{C}^{0}\left((0, T) \times \Gamma_{i}\right) \text { for } 1 \leq i \leq N\right\}
$$

The main result of this Note is summarized in the following theorem.

Theorem 2.2. Let $M \in \mathbb{N}^{*}$. Let $V_{M}$ be a subspace of $\mathcal{C}^{0}\left(0, T ; \mathcal{C}_{p c}^{0}\left(\Gamma_{\text {out }}\right)\right)$ spanned by some $M$ linearly independent functions and $K_{M}$ be any compact and convex subset of $V_{M} \cap L_{+}^{\infty}\left((0, T) \times \Gamma_{\text {out }}\right)$. Let $\Gamma \subseteq \Gamma_{0}$ be a nonempty open subset of the boundary, $u_{0} \in H^{1}(\Omega)$ be such that div $u_{0}=0$ in $\Omega$ and $g \in H^{1}\left(0, T ; L^{2}\left(\Gamma_{0}\right)\right)$ be such that $g(t)$ is not identically zero for all $t \in(0, T)$. Let $\left(u_{k}, p_{k}\right)$ be the weak solutions of system (1) with $q=q_{k} \in K_{M}$ for $k=1,2$. Then, there exists a constant $C>0$ such that

$$
\left\|q_{1}-q_{2}\right\|_{L^{\infty}\left((0, T) \times \Gamma_{\text {out }}\right)} \leq C\left\|u_{1}-u_{2}\right\|_{L^{2}((0, T) \times \Gamma)}
$$

The proof of Theorem 2.2 is based on an abstract theorem proved by L. Bourgeois in [6] that we recall in Theorem 3.4 and rely on the fact that the application which, to a Robin coefficient maps the velocity on $(0, T) \times \Gamma$ with $\Gamma \subseteq \Gamma_{0}$ is injective, of class $\mathcal{C}^{1}$ and its derivative is also injective. The result stated in [6] establishs Lipschitz stability estimates for a class of inverse problems. For instance, it allows to find again the stability results developed in [12] and [1] without resorting to quantification of unique continuation results. The author points out that one can find the original idea developed in [7] in the particular case of the detection of a moving obstacle in a fluid from measurements available on the boundary of the domain. Moreover, abstract theorems of the same type but with different assumptions can be found in [13]. The sequel of this paper is organized as follows. We present in Section 3 some preliminary results which will be useful to prove Theorem 2.2 . Then the proof of Theorem 2.2 is given in Section 4

\section{Preliminary results}

In the section, we state results which will be useful in the proof of Theorem 2.2. We begin by stating regularity result for a slightly more general Stokes system than system (1) (we add non homogeneous Robin boundary condition on $\left.(0, T) \times \Gamma_{\text {out }}\right)$ :

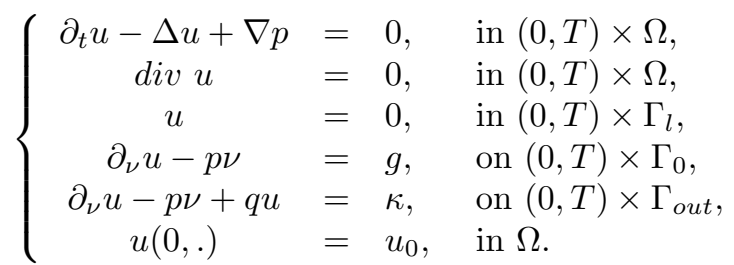


Proposition 3.1. Assume that $g \in H^{1}\left(0, T ; L^{2}\left(\Gamma_{0}\right)\right), \kappa \in H^{1}\left(0, T ; L^{2}\left(\Gamma_{\text {out }}\right)\right), u_{0} \in H^{1}(\Omega)$ be such that div $u_{0}=0$ in $\Omega$ and $q \in L_{+}^{\infty}\left((0, T) \times \Gamma_{\text {out }}\right)$. Then, system (2) has a unique solution which belongs to $L^{2}\left(0, T ; H^{1}(\Omega)\right) \cap H^{1}\left(0, T ; L^{2}(\Omega)\right) \times$ $L^{2}\left(0, T ; L^{2}(\Omega)\right)$. Moreover, there exists $C>0$, independent of $q$, such that the following inequality holds

$$
\|u\|_{L^{2}\left(0, T ; H^{1}(\Omega)\right)} \leq C\left(\left\|u_{0}\right\|_{L^{2}(\Omega)}+\|g\|_{L^{2}\left(0, T ; L^{2}\left(\Gamma_{0}\right)\right)}+\|\kappa\|_{L^{2}\left(0, T ; L^{2}\left(\Gamma_{\text {out }}\right)\right)}\right) .
$$

Proof of Proposition 3.1. The proof is mainly contained in the appendix of [5]. The main difference here is that we work with non homogeneous Robin boundary conditions which leads to slight modifications.

Remark 3.2. Note that due to the mixed boundary conditions, the fact that $\partial_{t} u \in E^{2}\left(0, T ; L^{2}(\Omega)\right)$ does not imply that $(u, p) \in L^{2}\left(0, T ; H^{2}(\Omega)\right) \times L^{2}\left(0, T ; H^{1}(\Omega)\right)$

The following Proposition 3.3 concerns the identifiability of the inverse problem we are interested in.

Proposition 3.3. Let $\Gamma \subseteq \Gamma_{0}$ be a nonempty open subset of the boundary, $u_{0} \in H^{1}(\Omega)$ be such that div $u_{0}=0$ in $\Omega$. Assume that $g \in H^{1}\left(0, T ; L^{2}\left(\Gamma_{0}\right)\right)$ is such that $g(t)$ is not identically zero for all $t \in(0, T)$. Let $\left(u_{k}, p_{k}\right)$ be the weak solutions of system (11) with $q=q_{k} \in \mathcal{C}^{0}\left(0, T ; \mathcal{C}_{p c}^{0}\left(\Gamma_{\text {out }}\right)\right)$ for $k=1,2$. We assume that $u_{1}=u_{2}$ on $(0, T) \times \Gamma$. Then $q_{1}=q_{2}$ on $(0, T) \times \Gamma_{\text {out }}$.

Proof of Proposition 3.3. The proof is based on the unique continuation result for the Stokes system proved by C. Fabre and G. Lebeau in [10]. Thanks to the previous proposition, $(u, p) \in L^{2}\left(0, T ; H^{1}(\Omega)\right) \cap H^{1}\left(0, T ; L^{2}(\Omega)\right) \times L^{2}\left(0, T ; L^{2}(\Omega)\right)$, which is enough regularity to prove a similar result to Corollary 3.2 in $[5]$. Then, we proceed exactly as in the proof of Proposition 3.3 in [5] where the proof is done in the particular case when the Robin coefficient does not depend on time, by arguing by contradiction.

As announced previously, the proof of our Lipschitz stability estimate is based on a theorem proved by L. Bourgeois in [6] which establishs Lipschitz stability estimate in an abstract framework for parameters defined on some finite dimensional subspace of the set of the continuous functions. For the sake of completeness, we state this theorem below.

Theorem 3.4. Let $\left(V,\|\|_{V}\right)$ and $\left(H,\|\|_{H}\right)$ be two Banach spaces. Let $U$ be an open subset of $V$ and $V_{M}$ a finite dimensional subspace of $V$ of dimension $M$. Let $K_{M}$ be a compact and convex subset of $V_{M} \cap U$. We consider a mapping $T: U \rightarrow H$ which satisfies the following assumptions:

1. $T: V_{M} \cap U \rightarrow H$ is injective,

2. $T: U \rightarrow H$ is $\mathcal{C}^{1}: T$ is differentiable in the sense of Fréchet at any point $x \in U$, the Fréchet derivative being denoted $d T_{x}: V \rightarrow H$ and the mapping $x \in U \rightarrow d T_{x} \in \mathcal{L}(V, H)$ is continuous.

3. For all $x \in V_{M} \cap U$, the operator $d T_{x}: V_{M} \rightarrow H$ is injective.

Then, there exists $C>0$ such that $\forall x, y \in K_{M},\|x-y\|_{V} \leq C\|T(x)-T(y)\|_{H}$.

\section{Proof of the main result}

In this section, we establishes the proof of Theorem 2.2. The proof consists of applying the abstract Theorem 3.4 with $V=L^{\infty}\left((0, T) \times \Gamma_{\text {out }}\right), H=L^{2}((0, T) \times \Gamma), U=L_{+}^{\infty}\left((0, T) \times \Gamma_{\text {out }}\right)$. We consider the operator

$$
\begin{aligned}
T: L_{+}^{\infty}\left((0, T) \times \Gamma_{\text {out }}\right) & \rightarrow L^{2}((0, T) \times \Gamma) \\
q & \left.\rightarrow u\right|_{\Gamma}
\end{aligned}
$$

where $(u, p)$ is solution of system (1). We are going to prove that:

1. $\left.T\right|_{V_{M} \cap U}$ is injective, 
2. $T$ is differentiable at any point $q \in L_{+}^{\infty}\left((0, T) \times \Gamma_{\text {out }}\right)$ and its Fréchet derivative is the operator

$$
d T_{q}: L^{\infty}\left((0, T) \times \Gamma_{\text {out }}\right) \rightarrow L^{2}((0, T) \times \Gamma)
$$

where $\left(v_{h}, \tau_{h}\right)$ is solution to

$$
\left\{\begin{array}{clll}
\partial_{t} v_{h}-\Delta v_{h}+\nabla \tau_{h} & =0, & & \text { in }(0, T) \times \Omega, \\
d i v v_{h} & =0, & & \text { in }(0, T) \times \Omega, \\
v_{h} & =0, & & \text { in }(0, T) \times \Gamma_{l}, \\
\partial_{\nu} v_{h}-\tau_{h} \nu & =0, & & \text { on }(0, T) \times \Gamma_{0}, \\
\partial_{\nu} v_{h}-\tau_{h} \nu+q v_{h} & =-h u, & & \text { on }(0, T) \times \Gamma_{\text {out }}, \\
v_{h}(0, .) & =0, & & \text { in } \Omega,
\end{array}\right.
$$

where $u$ is solution to system (1). Moreover, the mapping

$$
\begin{aligned}
d T: L_{+}^{\infty}\left((0, T) \times \Gamma_{\text {out }}\right) & \rightarrow \mathcal{L}\left(L_{+}^{\infty}\left((0, T) \times \Gamma_{\text {out }}\right), L^{2}((0, T) \times \Gamma)\right) \\
q & \rightarrow d T_{q}
\end{aligned}
$$

is continuous.

3. For all $q \in V_{M} \cap L_{+}^{\infty}\left((0, T) \times \Gamma_{\text {out }}\right)$, the operator $d T_{q}: V_{M} \rightarrow L^{2}((0, T) \times \Gamma)$ is injective.

Step 1 is a direct consequence of Proposition 3.3. Let us prove step 2. Let $q, h \in L_{+}^{\infty}\left((0, T) \times \Gamma_{\text {out }}\right)$ and $(u, p)($ resp. $\left.\left(u_{h}, p_{h}\right)\right)$ be the weak solution of system (1) associated to $q$ (resp. to $\left.q=q+h\right)$. We denote by $\left(w_{h}, \pi_{h}\right)=\left(u_{h}-u, p_{h}-p\right)$ which is solution of the following Stokes system:

$$
\left\{\begin{array}{clll}
\partial_{t} w_{h}-\Delta w_{h}+\nabla \pi_{h} & =0, & & \text { in }(0, T) \times \Omega, \\
d i v w_{h} & =0, & & \text { in }(0, T) \times \Omega, \\
w_{h} & =0, & & \text { in }(0, T) \times \Gamma_{l}, \\
\partial_{\nu} w_{h}-\pi_{h} \nu & =0, & & \text { on }(0, T) \times \Gamma_{0}, \\
\partial_{\nu} w_{h}-\pi_{h} \nu+q w_{h} & =-h u_{h}, & & \text { on }(0, T) \times \Gamma_{\text {out }}, \\
w_{h}(0, .) & =0, & & \text { in } \Omega .
\end{array}\right.
$$

Let $M_{1}>0$ be such that $\left\|u_{0}\right\|_{L^{2}(\Omega)}+\|g\|_{L^{2}\left(0, T ; L^{2}\left(\Gamma_{0}\right)\right)} \leq M_{1}$. Then, thanks to Proposition 3.1, there exists $C>0$ such that

$$
\left\|u_{h}-u\right\|_{L^{2}\left(0, T ; H^{1}(\Omega)\right)} \leq C\|h\|_{L^{\infty}\left((0, T) \times \Gamma_{\text {out }}\right)}\left\|u_{h}\right\|_{L^{2}\left(0, T ; H^{1}(\Omega)\right)} \leq C\left(M_{1}\right)\|h\|_{L^{\infty}\left((0, T) \times \Gamma_{\text {out }}\right)} .
$$

Now, let us consider $\left(e_{h}, \rho_{h}\right)=\left(u_{h}-u-v_{h}, p_{h}-p-\tau_{h}\right)$, where $\left(v_{h}, \tau_{h}\right)$ is solution to system (3). First, we readily check that the operator $\left.h \in L^{\infty}\left((0, T) \times \Gamma_{\text {out }}\right) \rightarrow v_{h}\right|_{\Gamma} \in L^{2}((0, T) \times \Gamma)$ is linear continuous. Secondly, $\left(e_{h}, \rho_{h}\right)$ solves the problem

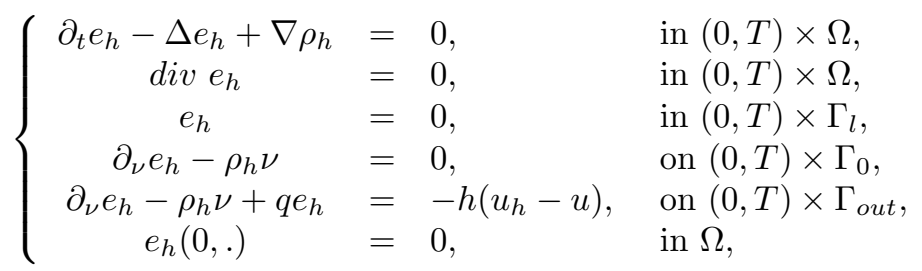

which implies, thanks to Proposition 3.1 and inequality ([5), that

$$
\left\|e_{h}\right\|_{L^{2}\left((0, T) ; H^{1}(\Omega)\right)} \leq C\|h\|_{L^{\infty}\left((0, T) \times \Gamma_{\text {out }}\right)}\left\|u_{h}-u\right\|_{L^{2}\left(0, T ; H^{1}(\Omega)\right)} \leq C\left(M_{1}\right)\|h\|_{L^{\infty}\left((0, T) \times \Gamma_{\text {out }}\right)}^{2},
$$

which proves that $T$ is Fréchet differentiable and $d T_{q}(h)=\left.v_{h}\right|_{\Gamma}$.

Let us prove now the continuity of the mapping $d T$ defined in (4). Let $\left(v_{h}, \tau_{h}\right)\left(\right.$ resp. $\left.\left(v_{h}^{l}, \tau_{h}^{l}\right)\right)$ be the solution of system (3) associated to $q$ (resp. $q=q+l)$ and where $(u, p)$ (resp. $(u, p)=\left(u_{l}, p_{l}\right)$ ) is the solution to system (11) associated to $q$ (resp. $q=q+l)$. We have that $\left(v_{h}^{l}-v_{h}, p_{h}^{l}-p_{h}\right)$ is the solution to the following Stokes system:

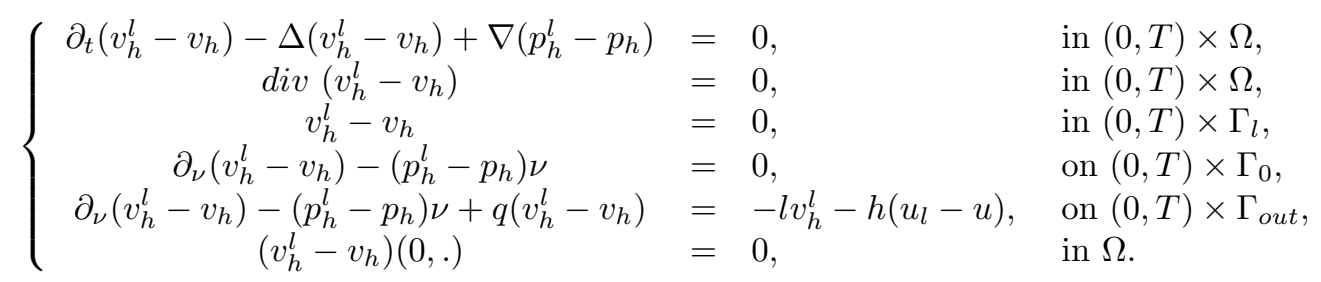


This implies, thanks to Proposition 3.1.

$$
\left\|v_{h}^{l}-v_{h}\right\|_{L^{2}\left(0, T ; H^{1}(\Omega)\right)} \leq C\|l\|_{L^{\infty}\left((0, T) \times \Gamma_{\text {out }}\right)}\left\|v_{h}^{l}\right\|_{L^{2}\left(0, T ; H^{1}(\Omega)\right)}+C\|h\|_{L^{\infty}\left((0, T) \times \Gamma_{\text {out }}\right)}\left\|u_{l}-u\right\|_{L^{2}\left(0, T ; H^{1}(\Omega)\right)},
$$

which leads to, applying again Proposition 3.1 and inequality (5) with $h=l$ :

$$
\left\|v_{h}^{l}-v_{h}\right\|_{L^{2}\left(0, T ; H^{1}(\Omega)\right)} \leq C\left(M_{1}\right)\|l\|_{L^{\infty}\left((0, T) \times \Gamma_{\text {out }}\right)}\|h\|_{L^{\infty}\left((0, T) \times \Gamma_{\text {out }}\right)},
$$

where $C$ is uniform with respect to $h$ and $l$. Otherwise, we have proved that

$$
\left\|d T_{q+l}-d T_{q}\right\|\|\leq C\| l \|_{L^{\infty}\left((0, T) \times \Gamma_{\text {out }}\right)},
$$

where ||| ||| denotes the operator norm. Thus the mapping $d T$ is continuous.

It remains to prove Step 3. Let $q \in L_{+}^{\infty}\left((0, T) \times \Gamma_{\text {out }}\right) \cap V_{M}$. Assume that $h \in V_{M}$ is such that $\left.v_{h}\right|_{(0, T) \times \Gamma}=0$. Then, since $\left(\left.v_{h}\right|_{(0, T) \times \Gamma},\left.\left(\partial_{\nu} v_{h}-\tau_{h} \nu\right)\right|_{(0, T) \times \Gamma)}=(0,0)\right.$, we obtain from unique continuation result that $v_{h}=0$ in $(0, T) \times \Omega$ and then $h u=0$ on $(0, T) \times \Gamma_{\text {out }}$. We conclude that $h=0$ by contradiction, exactly as for the injectivity of the mapping $T$ (see [5]).

\section{Bibliography}

[1] G. Alessandrini, E. Beretta, E. Rosset, and S. Vessella. Optimal stability for inverse elliptic boundary value problems with unknown boundaries. Ann. Scuola Norm. Sup. Pisa Cl. Sci. (4), 29(4):755-806, 2000.

[2] L. Baffico, C. Grandmont, and B. Maury. Multiscale modeling of the respiratory tract. Math. Models Methods Appl. Sci., 20(1):59-93, 2010.

[3] M. Bellassoued, J. Cheng, and M. Choulli. Stability estimate for an inverse boundary coefficient problem in thermal imaging. J. Math. Anal. Appl., 343(1):328-336, 2008.

[4] M. Boulakia, A.-C. Egloffe, and C. Grandmont. Unique continuation estimates for the Stokes system. Application to an inverse problem. Preprint, 2012.

[5] M. Boulakia, A.-C. Egloffe, and C. Grandmont. Stability estimates for a Robin coefficient in the two-dimensional Stokes system. Mathematical control and related field, 2(1), 2013.

[6] L. Bourgeois. A remark on lipschitz stability for inverse problems. Inria research report, RR-8104, Accepted for publication in Comptes Rendus de l'Académie des sciences, 2013.

[7] C. Conca, P. Cumsille, J. Ortega, and L. Rosier. On the detection of a moving obstacle in an ideal fluid by a boundary measurement. Inverse Problems, 24(4):045001, 18, 2008.

[8] A.-C. Egloffe. Étude de quelques problèmes inverses pour le système de Stokes. Application aux poumons. PhD thesis, Université Paris VI, 2012.

[9] A.-C. Egloffe. Lipschitz stability estimate in the inverse Robin problem for the Stokes system. Inria research report, $R R$-8222, 2013.

[10] C. Fabre and G. Lebeau. Prolongement unique des solutions de l'equation de Stokes. Comm. Partial Differential Equations, 21(3-4):573-596, 1996.

[11] A. Quarteroni and A. Veneziani. Analysis of a geometrical multiscale model based on the coupling of ODEs and PDEs for blood flow simulations. Multiscale Model. Simul., 1(2):173-195 (electronic), 2003.

[12] E. Sincich. Lipschitz stability for the inverse Robin problem. Inverse Problems, 23(3):1311-1326, 2007.

[13] P. Stefanov and G. Uhlmann. Linearizing non-linear inverse problems and an application to inverse backscattering. J. Funct. Anal., 256(9):2842-2866, 2009.

[14] I. E. Vignon-Clementel, C. A. Figueroa, K. E. Jansen, and C. A. Taylor. Outflow boundary conditions for threedimensional finite element modeling of blood flow and pressure in arteries. Comput. Methods Appl. Mech. Engrg., 195(29-32):3776-3796, 2006. 